MATEC Web of Conferences 33, 04003 (2015)

DOI: $10.1051 /$ matec conf/ 20153304003

(C) Owned by the authors, published by EDP Sciences, 2015

\title{
An Experimental Study on Rate-sensitive Tensile Deformation Behaviour of Fe-based Shape Memory Alloy
}

\author{
Takeshi IWAMOTO ${ }^{1, a}$ and Kazuki FUJITA \\ ${ }^{1}$ Institute of Engineering, Hiroshima University, 1-4-1 Kagamiyama, Higashi-Hiroshima, Hiroshima, 739-8527 Japan \\ ${ }^{2}$ Graduate School of Engineering, Hiroshima University, 1-4-1 Kagamiyama, Higashi-Hiroshima, Hiroshima, $739-8527$ Japan
}

\begin{abstract}
Recently, it is attempted to apply high manganese steel including Fe-based shape memory alloy to vibration dampers. Especially, the alloy indicates a special characteristic as a well-known shape memory effect. By coupling between this effect and its plastic deformation, it can be considered that its deformation behaviour at higher deformation rate becomes quite complicated and still unclear. In this study, tensile tests of Fe-based shape memory alloy at different rate of deformation are conducted by using two different testing apparatuses such as the conventional material testing machine and impact testing machine based on the split Hopkinson pressure bar technique. In the tests, temperature rise is captured during the quasi-static deformation. After the quasi-static test, the recovery strain due to shape memory effect is measured by heating up the deformed specimens to $A_{f}$ temperature. Finally, the rate sensitivity of the alloy is discussed including the recovery strain.
\end{abstract}

\section{Introduction}

Recently, it is attempted to apply high manganese steels to vibration dampers with high performance. It is clarified that the highly damping property can be obtained by controlling the chemical composition. Various kinds of excellent steel such as Twinninginduced Plasticity (TWIP) steel, Fe-based shape memory alloy (SMA), etc. are included into the category of high manganese steels. These steels are attracting the interest of not only industries but also the scientific community.

Among various kinds of SMAs, the shape memory effect (SME) of Fe-based SMA [1] (Fe-SMA) is lower than that of other SMAs; however, it shows the excellent formability, machinability, weldability [2], etc. In addition, its production cost is cheaper than other SMAs [3]; therefore, the alloy is attempted to be applied to large-scale structural members [4] such as the joint in the pipeline [5]. A pipe joint made of Fe-SMA has already been used practically for a construction of a tunnel [6].

When Fe-based SMA is used as the members, it is conceivable to deform at higher strain rate because of earthquake or wind load. A clarification of the strength under axial tensile deformation in the members at various strain rate from quasi-static to impact test is strongly required for designing safety and reasonable structures. Therefore, it is important to investigate the rate sensitivity of the Fe-based SMA. From this point of view, Ogawa and Yamada [7] investigate the rate sensitivity and temperature dependence of tensile deformation behaviour in Fe-14Mn-6Si-9Cr-5Ni alloy by using both the material testing machine and impact testing machine based on split Hopkinson pressure bar (SHPB) technique. As the result, they reported that positive rate sensitivity as similar to the metallic materials can be seen. However, the almost constant SME can be expected in the any range of strain rate. By coupling between the SME and its plastic deformation, it can be considered that its deformation behaviour at higher strain rate becomes quite complicated and still unclear.

In this study, tensile tests of Fe-based SMA at different strain rates are conducted by two different testing apparatuses such as the conventional material testing machine and impact testing machine based on SHPB technique. In the tests, temperature rise is captured during the quasi-static deformation by a type $\mathrm{T}$ thermocouple. After the quasi-static test, the recovery strain due to shape memory effect is measured by heating up the deformed specimens to $A_{f}$ temperature. Finally, the rate sensitivity of the alloy is discussed including the recovery strain.

\section{Experimental Procedure}

\subsection{Material and specimens}

The Fe-based SMA used in this study contains four principal elements such as $\mathrm{Fe}, \mathrm{Mn}, \mathrm{Si}$ and $\mathrm{Cr}$. The $\mathrm{Fe}$ $28 \mathrm{Mn}-6 \mathrm{Si}-5 \mathrm{Cr}$ alloy commercially supplied by Awaji Materia Co., Ltd is the most widely used due to its properties. Table 1 shows the chemical composition of the alloy (mass\%). According to the catalogue of this

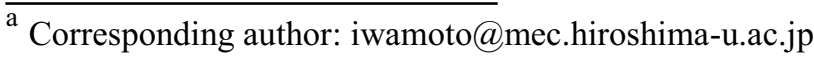


alloy, $\mathrm{M}_{\mathrm{s}}$ temperature is 253 to $298 \mathrm{~K}$ and $\mathrm{A}_{\mathrm{f}}$ temperature is 403 to $458 \mathrm{~K}$, respectively.

Table 1. Chemical composition of the alloy used here (mass\%).

\begin{tabular}{|c|c|c|c|c|c|}
\hline \hline $\mathrm{C}$ & $\mathrm{Si}$ & $\mathrm{Mn}$ & $\mathrm{P}$ & $\mathrm{S}$ & $\mathrm{Cr}$ \\
\hline 0.01 & 6.06 & 28.15 & 0.003 & 0.013 & 4.82 \\
\hline \hline $\mathrm{N}$ & $\mathrm{Ni}$ & \multicolumn{4}{l}{} \\
\cline { 1 - 2 } 0.009 & 0.04 & &
\end{tabular}

The specimen made of the alloy is manufactured by machining. Fig. 1 shows photographs and geometries of specimens for (a) quasi-static and (b) impact tests, respectively. For the quasi-static test, dimensions are 7 $\mathrm{mm}$ in diameter and $45 \mathrm{~mm}$ in a gauge length. On the other hand, $4 \mathrm{~mm}$ in diameter and $4 \mathrm{~mm}$ in a gauge length are chosen for impact test. The specimens of Fe-SMA after machining is quenched into cold water after it is heated to $1223 \mathrm{~K}$ for 30 minutes by an electric furnace. As the result of this heat treatment called shape memory treatment, residual stress caused by machining can be removed.

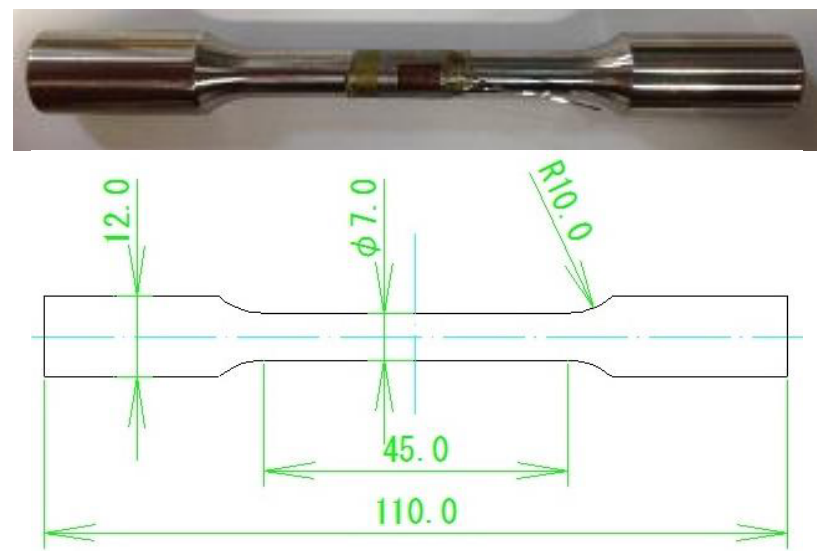

(a) For quasi-static tension

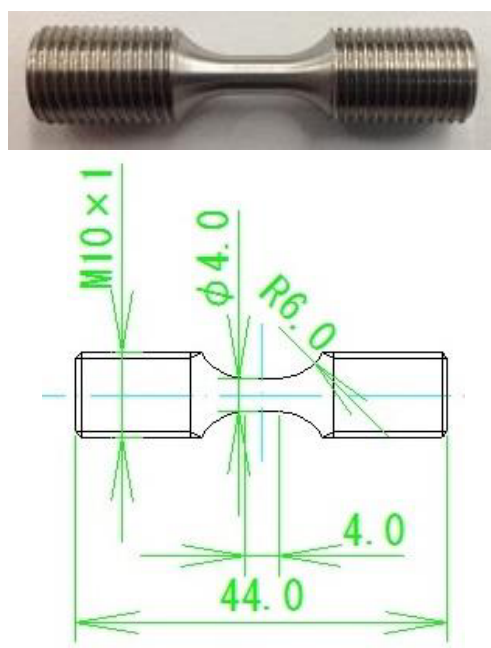

(b) For impact tension

Figure 1. Photographs and drawings of specimens used here.

\subsection{Testing apparatuses and methods}

Quasi-static tensile test is carried out with an INSTRONtype material testing machine (Shimadzu AG-250KN). Strain rate is controlled by a speed of the crosshead equipped up to the 0.02 of nominal strain. As a result, constant strain rates as $10^{-4}, 10^{-3}, 10^{-2}$ and $10^{-1} / \mathrm{s}$ are obtained. As shown in Fig.1 (a), the strain gauge is glued at the centre of the specimens. After unloading, the specimen is placed in the electric furnace, heated up to $523 \mathrm{~K}$ for $30 \mathrm{~min}$ and then furnace-cooled under natural convection. Theoretically speaking, the temperature in this recovery process is quite higher than $\mathrm{A}_{\mathrm{f}}$ temperature. Thus, it can be considered that martensitic phase fully transforms to the parent phase. Recovery strain is measured by glued strain gage. Recovery strain is defined as the axial strain decreasing with respect to environmental temperature change in the furnace. Thus, it has a value of 0 in the initial. Additionally, temperature is recorded by a type $\mathrm{T}$ thermocouple fixed on the surface of the specimen during tensile deformation.

Impact tensile test is conducted by using an apparatus based on the split Hopkinson pressure bar method (SHPB). Fig. 2 shows the schematic diagram of the impact tensile testing apparatus based on the SHPB technique used in this experiment. The tensile incident, the compressive reflected and the tensile transmitted stress waves are recorded by the two strain gauges glued on the pressure bars with full bridge method, and impact stress-strain curve of the specimen can be calculated by the theory of stress wave propagation in one-dimensional elastic bar. Here, the material of the stress bar is the bearing steel (SUJ2 in JIS) and the diameter of the pressure bars is $16 \mathrm{~mm}$. Both input and output bars have $3000 \mathrm{~mm}$ and $1300 \mathrm{~mm}$ in length, respectively. Striker tube with $500 \mathrm{~mm}$ in length has $16 \mathrm{~mm}$ and $23 \mathrm{~mm}$ in inner and outer diameters. By controlling the air pressure in the tank, three different impact velocities of the striker tube are obtained. The impact velocities are 4.7, 9.7 and $11.4 \mathrm{~m} / \mathrm{s}$, respectively.

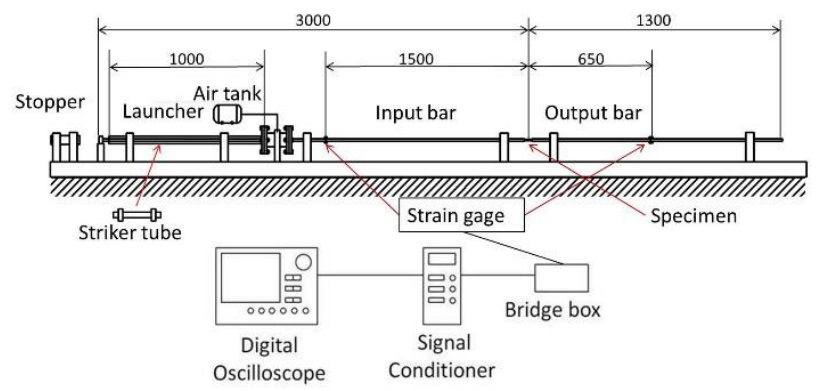

Figure 2. Schematic illustration of the split Hopkinson pressure bar apparatus for impact tension.

When the striker tube impacts onto the input bar, incident stress pulse propagates through in the input bar. After that, a part of the incident pulse propagates to in the output bars as transmitted pulse via vending deformation of the specimen. The other part of the incident pulse propagates back into the input bar as reflected pulse. Using these measured pulses and one dimensional wave propagation theory, load applied to the specimen, deflection and deflection rate can be calculated. By 
processing incident stress pulse $\sigma_{i}(t)$, reflected stress pulse $\sigma_{r}(t)$ and transmitted stress pulse $\sigma_{t}(t)$, nominal strain rate $\dot{\varepsilon}(t)$, nominal strain $\varepsilon(t)$ and nominal stress $\sigma(t)$ are obtained by the followed equations

$$
\begin{gathered}
\dot{\varepsilon}(t)=-\frac{2}{\rho c l} \sigma_{r}(t), \\
\varepsilon(t)=\int_{0}^{t} \dot{\varepsilon}\left(t^{\prime}\right) d t^{\prime}, \text { and } \\
\sigma(t)=\frac{A_{B}}{A} \sigma_{t}(t) .
\end{gathered}
$$

where $\rho$ is mass density and $c$ is a velocity of longitudinal elastic wave propagation in pressure bars. $A$ and $A_{B}$ are cross-sectional areas of specimen and pressure bars.

\section{Experimental procedures}

\subsection{Rate sensitivity of tensile deformation during loading process}

Figure 3 shows the time history of nominal strain rate during impact tensile deformation by SHPB technique at different impact velocities of the striker tube. From this figure, the almost constant strain rate can be obtained. In addition, the duration of these curve is almost the same. The values of the plateau region in the time history of strain rates becomes 1000, 2250 and 2600, respectively.

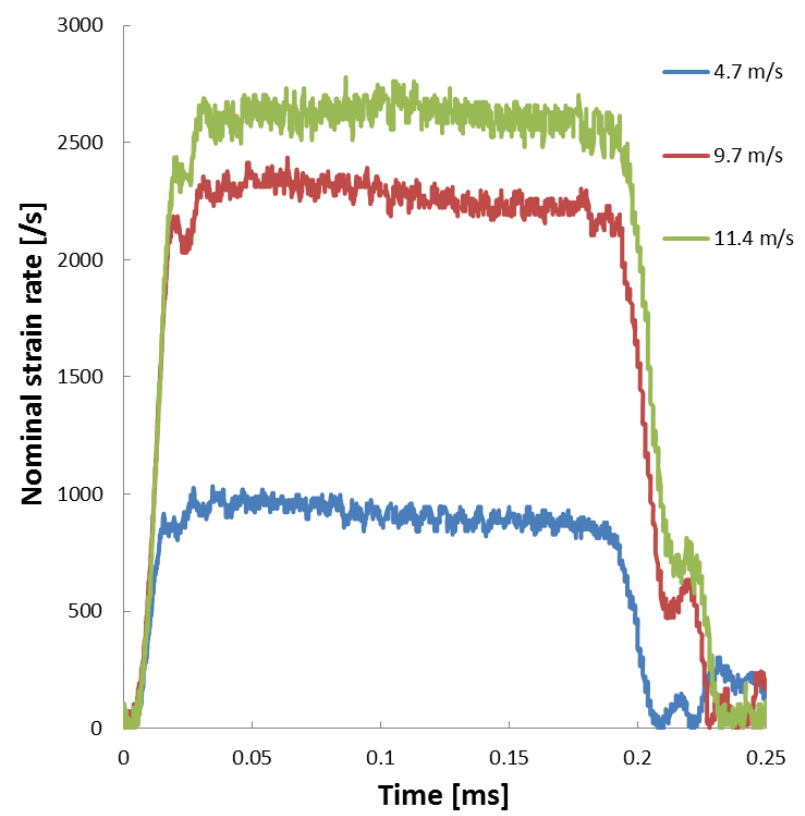

Figure 3. Time history of nominal strain rate during impact tensile deformation by SHPB technique at different impact velocities of the striker tube.

Fig. 4 shows true stress vs true strain at various strain rate. As shown in this figure, the curves at all strain rates indicates concave and the shape of the curves is quite similar to that of the conventional metallic materials which the plastic deformation occurs. The positive rate sensitivity can be observed in the entire region of true strain. The positive rate sensitivity is defined that the level of true stress increases with increasing the nominal strain rate. This tendency can be also observed in the conventional metallic materials.
Fig. 5 shows a semi-logarithmic plot of the relationship between true stress at 0.01 of true strain and nominal strain rate. As shown in this figure, the true stress at 0.01 of true strain is linearly increasing with respect to the nominal strain rate. Clear rate sensitivity on the true stress can be seen. Therefore, the consideration of this sensitivity is indispensable to design structures or materials which the higher speed of deformation such as the damper.

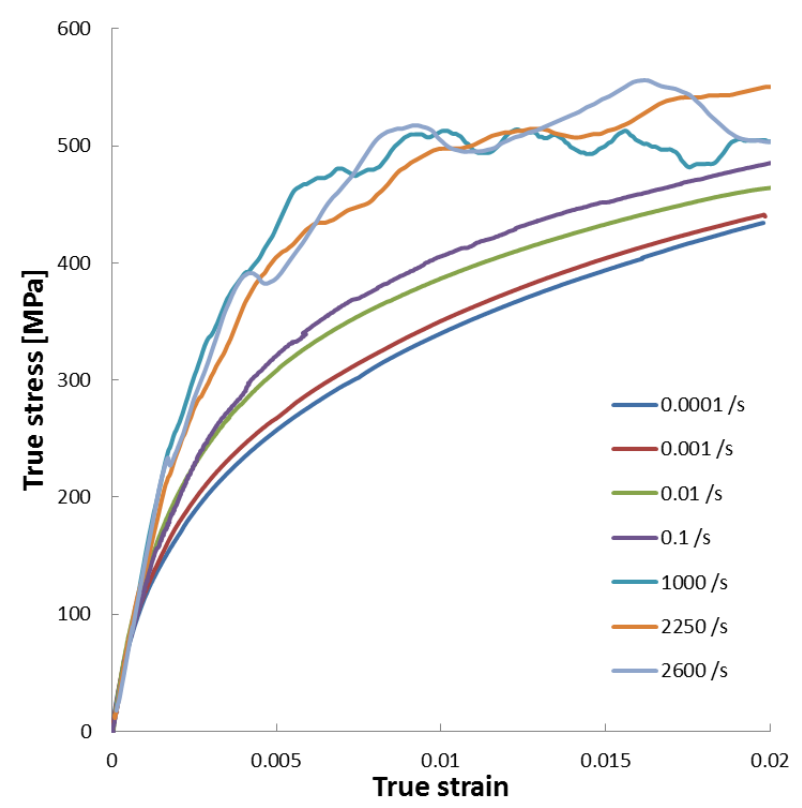

Figure 4. Relationship between true stress and true strain at various strain rate.

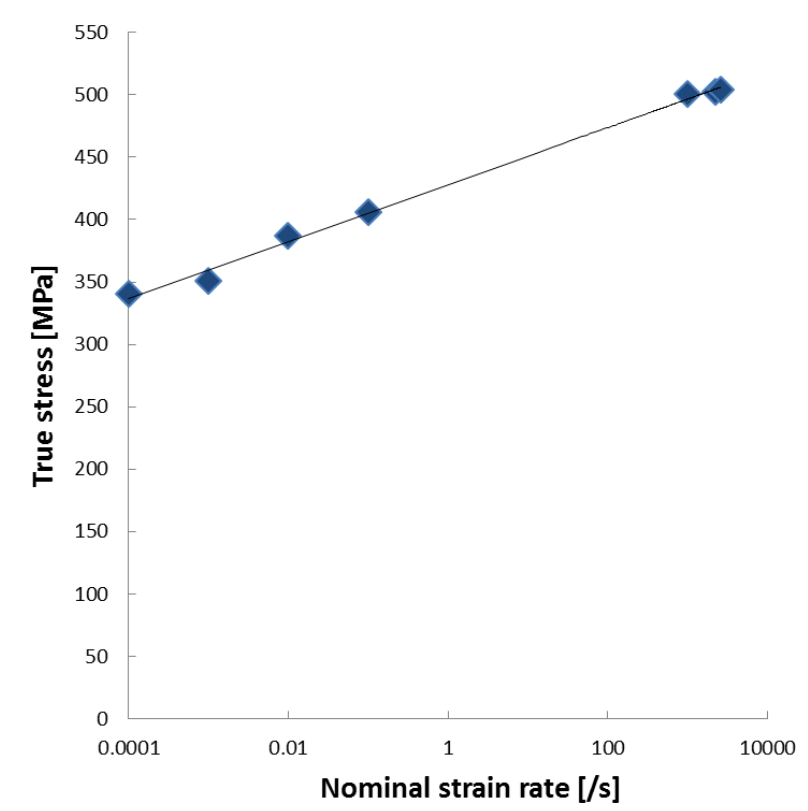

Figure 5. A semi-logarithmic plot of the relationship between true stress at 0.01 of true strain and nominal strain rate.

\subsection{Rate sensitivity during recovery process}

Figure 6 shows time history of recovery strain during heating after tensile deformation at various strain rates. The time in this figure is normalized by the maximum 
time of recovery process. Initially, the recovery strain increases because of thermal expansion, however, the amount is quite small. After that, the gradual decrease in the strain can be observed. After about 0.4 of normalize time, recovery strain becomes constant for all conditions. When the rate sensitivity of recovery strain is focused upon, the final value of recovery strain decreases with an increase in the nominal strain rate.

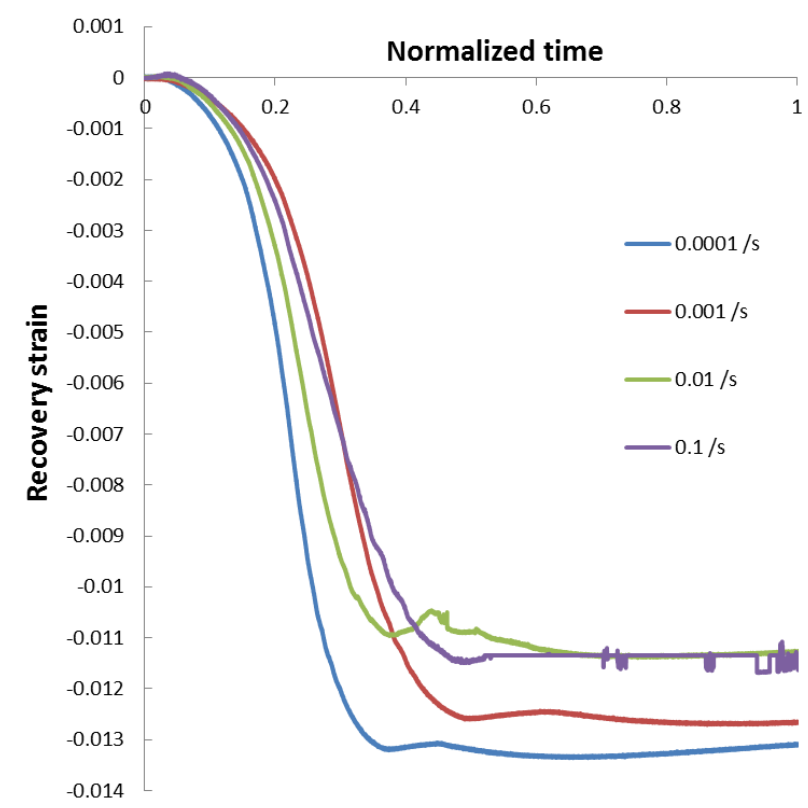

Figure 6. Time history of axial strain during heating after tensile deformation.

Fig. 7 shows a ratio of shape recovery defined by Inagaki [8] and the absolute values of measured strain vs nominal strain rate. The definition of the ratio of shape recovery is given by the ratio between the total residual strain after unloading and the recovery strain [8]. Blue diamonds and line indicates the ratio of shape recovery and its approximated line respect to the strain rate. As shown in this figure, the ratio of shape recovery slightly decreases with nominal strain rate. However, it can be considered that this is almost constant because the slope is quite small. As the result, the SME against different residual strain is independent from the strain rate. In the figure, the red square and green triangle indicates the residual strain measured just after unloading and recovery strain measured just after recovery process. Red and green lines show their approximated lines and their colours corresponds to those of symbols. Absolute values of these strain decrease with increasing the strain rate. If these slopes of these lines are the same, the ratio of shape recovery constantly changes with the strain rate. However, the slopes become different and the slope of the line for the recovery strain is slightly steeper than the other. As a result, the ratio of shape recovery decreases.

\subsection{Temperature rise under quasi-static tension}

Figure 8 shows temperature rise vs normalized time measured during quasi-static tension. In this figure, time is normalized by the time when temperature indicates its maximum value during the quasi-static deformation. In the initial, the quite small negative temperature rise can be observed for all the conditions of strain rate. It can be considered that the thermo-elastic effect occurs. In the case of the highest strain rate, the maximum temperature rise is about $4.7 \mathrm{~K}$. With decreasing strain rate, this maximum value decreases. Comparing with the other materials, the temperature rise is quite small.

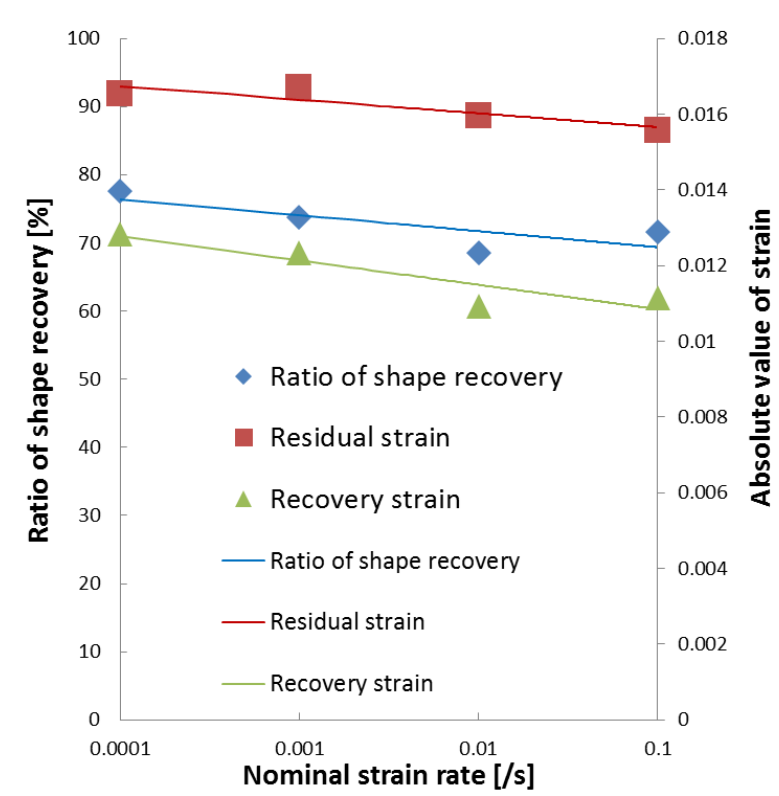

Figure 7. Changes in the ratio of shape recovery, residual strain and recovery strain with respect to nominal strain rate in the range of quasi-static deformation.

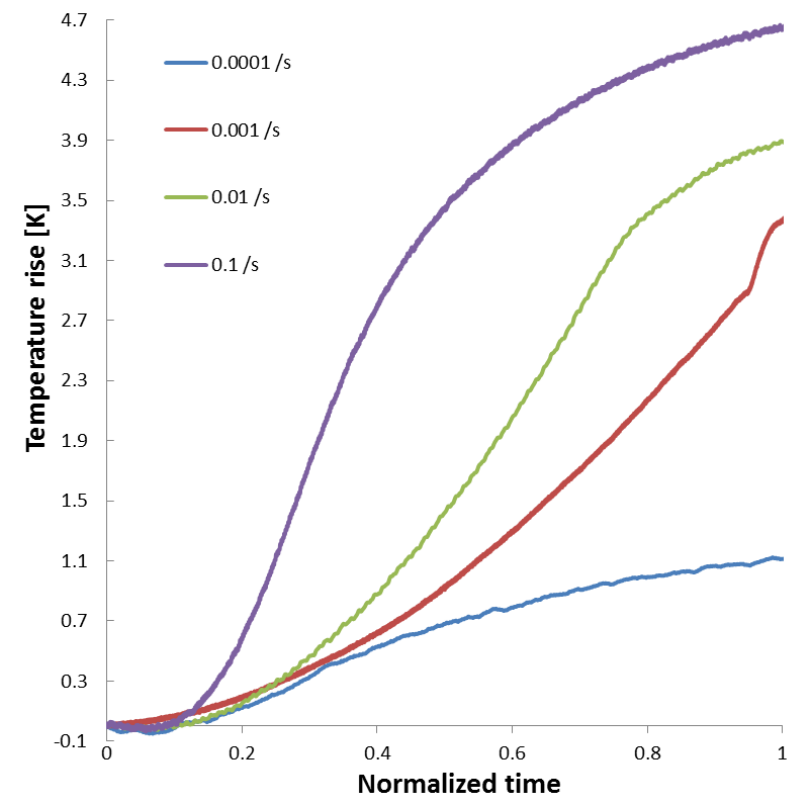

Figure 8. Temperature rise vs normalized time measured during quasi-static tension.

\section{Discussions}


In order to discuss the validity of experimental results of temperature, the heat conduction equation is considered as follows [9]

$$
\dot{T}=\frac{\zeta}{\rho C_{v}} \bar{\sigma} \dot{\bar{\varepsilon}}^{i n}+\frac{\kappa}{\rho C_{v}} \nabla^{2} T+\frac{\ell^{\varepsilon}}{C_{v}} \dot{f}^{\varepsilon},
$$

where $\bar{\sigma}$ is equivalent stress, $\dot{\bar{\varepsilon}}^{\text {in }}$ is equivalent inelastic strain, $f^{\varepsilon}$ is volume fraction of martensite, $T$ is absolute temperature, $\zeta$ is Taylor-Quinney coefficient [10], $\kappa$ is the thermal conduction coefficient, $\ell^{\varepsilon}$ is transformation latent heat, $C_{v}$ is specific heat at constant volume and ( ) is the material time derivative of ( ). Normally, $\zeta$ takes value of 0.85 to 0.95 for metallic materials and its physical meaning is the rate of inelastic work which can be converted into heat [10]. Thus, the rest of inelastic work denoted by $1-\zeta$ is stored in the material such as the thermal activation process of dislocations. If the first term of right hand side in Eq. (4) including strain rate becomes extremely-larger than the other terms and rate of heat transfer, temperature rise $\Delta T$ can be calculated as

$$
\Delta T=\frac{\zeta}{\rho C_{v}} \int_{0}^{\varepsilon_{\max }} \bar{\sigma} d \bar{\varepsilon}^{i n} .
$$

As shown in Fig. 7, the plastic deformation is quite small so that it can be considered that $\xi$ becomes almost unit. After calculating the integral in Eq. (5) from Fig. 4, $\Delta T$ in the quasi-static tension can be obtained as shown in Table 2 .

Table 2. Comparison of temperature rise obtained by experiment and calculation using Eq. (5).

\begin{tabular}{|c|c|c|}
\hline Strain rate $[/ \mathrm{s}]$ & $\begin{array}{c}\Delta T[\mathrm{~K}] \\
\text { (Eq. (5)) }\end{array}$ & $\begin{array}{c}\Delta T[\mathrm{~K}] \\
\text { (Experiment) }\end{array}$ \\
\hline \hline $10^{-4}$ & 1.39 & 1.11 \\
\hline $10^{-3}$ & 1.44 & 3.36 \\
\hline $10^{-2}$ & 1.62 & 3.88 \\
\hline $10^{-1}$ & 1.67 & 4.64 \\
\hline
\end{tabular}

In the calculation, $5.47 \times 10^{-4} \mathrm{~J} / \mathrm{kg} \mathrm{K}$ and $7454 \mathrm{~kg} / \mathrm{m}^{3}$ for $C_{v}$ and $\rho$ are used from the catalogue. $\Delta T$ evaluated by Eq. (4) decreases if the second and thrid terms in right hand side are smaller than the first term. In addition, similar tendency can be shown when the rate of heat transfer vanished in Eq. (5) is quite faster than strain rate. As shown in Table 2, a quantitative correspondence between the obtained values of $\Delta T$ by theory and experiment cannot be shown. In the case of $10^{-4} / \mathrm{s}$, theoretical $\Delta T$ is larger than experimental result. For slower deformation, of course, the assumptions cannot be satisfied since heat transfer is dominant. With increasing the strain rate, this tendency becomes opposite. This means the heat transfer becomes smaller and the effect of latent heat becomes dominant for higher speed of deformation. Totally, the quantitative difference between these values of $\Delta T$ and its rate sensitivity are accountable. Therefore, it can be considered that the measured value of $\Delta T$ is qualitatively reasonable.

As shown in Fig. 5, it can be considered that the rate sensitivity in this alloy is not small. Therefore, a consideration of the sensitivity may be important to design structures using Fe-based SMA subjected to the impact deformation. Before that, let discuss about the mechanism of the sensitivity of tensile deformation behaviour during loading process. From Figs. 4 and 5, it is possible that the mechanism of the sensitivity is fully plastic deformation because the shape of the curve and tendency are quite similar to the conventional metallic materials. However, it may be found that this direction of discussions will induce mistakes when Fig. 7 is looked at and this alloy shows the SME because of stress-induced martensitic transformation. For the sensitivity, three possible mechanisms and their combination can be considered as follows.

1. Plastic deformation in the parent phase

$$
\begin{aligned}
& \text { i. } \quad \text { Corresponding to the applied external force } \\
& \text { ii. Accommodation process for transformation } \\
& \text { strain }
\end{aligned}
$$

2. Self-heating by the irreversible work of deformation and transformation latent heat

3. Martensitic transformation itself

According to the mechanism \#1, stress increases with respect to the strain rate as the famous thermal activation process because of the viscous drag for mobile dislocation and/or twinning. As shown in Fig. 8, temperature increases with the promotion of the deformation. The temperature rise induces the suppression of martensitic transformation. To achieve the required level of strain, higher stress should be applied because of the suppression. In order to explain the mechanism \#3, let consider an ideal situation without the heating due to irreversible work. If martensitic transformation itself will becomes lower with respect to the strain by changing in the strain rate, much higher stress is required in the deformation.

From Fig. 7, the recovery strain decreases with respect to the strain rate. If the strain is generated by martensitic transformation, the amount of martensite decreases with respect to the strain rate. Additionally, temperature effect is quite important to reduce the amount of martensite in the alloy. As above-mentioned, $\mathrm{M}_{\mathrm{s}}$ temperature is near the room temperature. It will be sufficient to supress the martensitic transformation partially even if $1 \mathrm{~K}$ of temperature increases from the results of Fig. 8. As a result, it becomes significant to increase the level of true stress. Of course, their combination should be considered. Especially, the plastic deformation in the parent phase due to transformation strain is dominant in the initial of deformation.

\section{Concluding Remarks}

In this study, tensile tests of Fe-based SMA at different strain rates were conducted by using two different testing apparatuses. In the tests, temperature rise was captured during the quasi-static deformation by thermocouple. After the quasi-static test, the recovery strain due to shape memory effect is measured by heating up the deformed specimens to $\mathrm{A}_{\mathrm{f}}$ temperature. The following results can be obtained.

1. True stress increases with increasing the strain rate. The positive rate sensitivity on the deformation behaviour can be clearly observed. 
2. In the quasi-static test with different strain rate, the ratio of shape recovery is independent from the strain rate.

\section{References}

1. A. Sato, E. Chishima, K. Soma, T. Mori, Acta Metall. 30, 1177 (1982)

2. W. Khalil, L. Saint-Sulpice, S. Arbab Chirani, C. Bouby, A. Mikolajczak, Mech. of Mater. 63, 1 (2013)

3. F. Nishimura, N. Watanabe, K. Tanaka, Comp. Mater. Sci. 8, 349 (1997)
4. M. Wada, H. Naoi, T. Maruyama, Proc. CAMP-ISIJ 17, 1044 (2004) (in Japanese)

5. J. C. Li, X. X. Lü, Q. Jiang, ISIJ Int. 40, 1124 (2000)

6. T. Kasuya, T. Obata, H. Miki, T. Maruyama, Proc. Symp. Underground Space 7, 371 (2002) (in Japanese)

7. K. Ogawa, A. Yamada, Zairyo - J. Soc. Mater. Sci. Jpn. 40, 844 (1991) (in Japanese)

8. H. Inagaki, Z. Metallkd. 83, 90(1992)

9. T. Tomita, T. Iwamoto, Int. J. Mech. Sci. 37, 1295 (1995)

10. G. Taylor, H. Quinney, Proc. R. Soc. Lond. A 143, 307 (1934). 